\title{
Effect of various levels of nitrogen and phosphorus on nutrient content and uptake of Palmarosa (Cymbopogan martinii var. Motia)

\author{
M. JAYALAKSHMI ${ }^{1}$, S.G. WANKHADE ${ }^{2}$, P. MOHAN RAO ${ }^{3}$ AND D.G.M. SAROJA ${ }^{4}$ \\ ${ }^{184}$ Central Research Institute for Dryland Agriculture, Hyderabad-500 059 (AP), India \\ ${ }^{2}$ Dr.Panjab Rao Deshmukh Krish Vidyapeeth, Akola-444 104(MS), India \\ ${ }^{3}$ Acharya N.G Ranga Agricultural Universit, Rajendranagar, Hyderabad-500 030(AP), India \\ E-mail: jayalakshmimitnala@gmail.com
}

\begin{abstract}
A field experiment was conducted to study the different levels of Nitrogen and Phosphorus on nutrient content and uptake of Palmarosa under rainfed conditions on vertisols at Panjabrao Deshmukh Krishi Vidyapeeth, Akola during 2007-2008. The experiment was laid in factorial randomized block design with three replications. There were four levels of nitrogen viz., $0 \mathrm{k} \mathrm{g}\left(\mathrm{N}_{0}\right), 40 \mathrm{~kg}\left(\mathrm{~N}_{1}\right), 60 \mathrm{~kg}\left(\mathrm{~N}_{2}\right), 80$ $\mathrm{kg}\left(\mathrm{N}_{3}\right)$ and three levels of phosphorus $0 \mathrm{~kg}\left(\mathrm{P}_{0}\right), 20 \mathrm{~kg}\left(\mathrm{P}_{1}\right), 40 \mathrm{~kg}\left(\mathrm{P}_{2}\right) \mathrm{ha}^{-1}$. The results revealed that the nitrogen content $(0.85 \%)$, phosphorus content $(0.69 \%)$ and potassium content $(0.56 \%)$ in the crop was

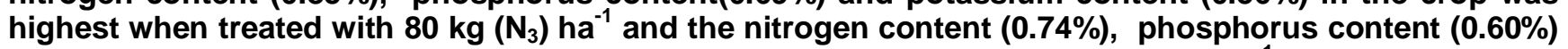

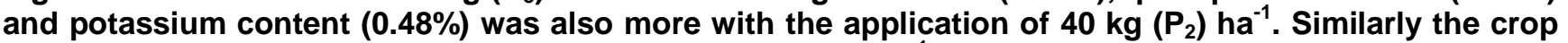
uptake was recorded highest with application of $80 \mathrm{~kg} \mathrm{~N} \mathrm{ha}^{-1}$ where the nitrogen uptake was $(30.50 \mathrm{~kg}$ $\left.\mathrm{ha}^{-1}\right)$, phosphorus uptake $\left(14.36 \mathrm{~kg} \mathrm{ha}^{-1}\right)$ and potassium uptake $\left(13.25 \mathrm{~kg} \mathrm{ha}^{-1}\right)$, while the nitrogen uptake $\left(22.12 \mathrm{~kg} \mathrm{ha}^{-1}\right)$, phosphorus uptake $\left(11.90 \mathrm{~kg} \mathrm{ha}^{-1}\right)$ and potassium uptake $\left(10.12 \mathrm{~kg} \mathrm{ha}^{-1}\right)$ was also more when treated with $40 \mathrm{~kg}\left(\mathrm{P}_{2}\right)$ ha. ${ }^{-1}$
\end{abstract}

Key words: Content, Nutrient uptake and Palmarosa,

Palmarosa (Cymbopogan martini var. Motia) commonly known as Rosha grass is a tall perennial tufted hedge native of most parts of sub-tropical India. Palmarosa oil has a sweet floral rose-like odour also has notes of rye bread, tea and clary sage (Mallavarapu et al., 1998). The oil is extensively used as perfumery raw material in soaps; floral rose-like perfumes; cosmetics preparations and in the manufacture of mosquito repellent products. It is used for flavoring tobacco products, foods and non-alcoholic beverages (Prakasa Rao et al., 1985). The volatile oil is used as a remedy for lumbago, stiff joints, skin diseases and for bilious complaints (Rajeswara Rao, 1999). The crop is estimated to remove $31.6,74$ and $75 \mathrm{~kg}$ each of NPK from the soil besides $34.8 \mathrm{~kg} \mathrm{Ca}, 20.6 \mathrm{~kg} \mathrm{Mg}, 0.9$ and $0.034 \mathrm{~kg}$ of $\mathrm{Mn}$ and $\mathrm{Zn}$ respectively in a harvest of $108 \mathrm{q}$ ha $^{-1}$ of dry mass (Pareek et al., 1983).

It is universally accepted that the use of chemical fertilizers is an integral part of the package of practices (like use of improved seeds, proper soil and water management, improved cultural practices, plant protection measures, post harvest operations etc.) for raising the production to a higher one. Studies conducted by the FAO have established beyond doubt that there is close relationship between the overuse crop yields and fertilizer consumption level. The economy return from Palmarosa plantation mainly depend upon the proper selection of variety, its nutritional management, the biomass yield, percentage of oil in the herbage and quality of the oil. Studies on the nutrient content, and uptake of a crop helps in judging the efficacy of applied nutrient elements through fertilizers and it is directly correlated in the biomass yield. The content of nutrients also indicates quality of oil produced. The present work was therefore undertaken to study the periodic concentration and uptake of nutrients by Palmarosa crop under recommended cultivation practices and different nitrogen and phosphorus fertility levels.

\section{MATERIALS AND METHODS}

A field experiment was conducted at Nagarjun Medicinal and Aromatic Plants Garden, Dr. Panjabrao Deshmukh Krishi Vidyapeeth Akola during Kharif 20072008. The soil site selected for the experiment was medium black and medium fertility status with good drainage. There were twelve treatment combinations laid in factorial randomized block design with three replications. The treatment include four levels of nitrogen viz., $0 \mathrm{~kg}\left(\mathrm{~N}_{0}\right), 40 \mathrm{~kg}\left(\mathrm{~N}_{1}\right), 60 \mathrm{~kg}\left(\mathrm{~N}_{2}\right), 80 \mathrm{~kg}\left(\mathrm{~N}_{3}\right)$ and three levels of phosphorus $0 \mathrm{~kg}\left(\mathrm{P}_{0}\right), 20 \mathrm{~kg}\left(\mathrm{P}_{1}\right), 40 \mathrm{~kg}\left(\mathrm{P}_{2}\right) \mathrm{ha}^{-1}$. Four weeks old seedlings were planted at the spacing of $45 \times 30 \mathrm{~cm}$. Protective irrigations were given at timely interval as and when required. Nitrogen was applied through urea in three split doses as per 2:1:1 basal dose, after $1^{\text {st }}$ cutting and after $2^{\text {nd }}$ cutting respectively. Phosphorus was applied as per the treatment as basal dose at the time of planting through single super phosphate. Potassium was applied as basal dose @ $40 \mathrm{~kg} \mathrm{ha}^{-1}$ through murate of potash to all the treatments. The first cutting was done after 110 days of planting i.e. at flowering stage, subsequently second and third cuttings were undertaken after 90 and 170 days after $1^{\text {st }}$ cutting respectively. The second cutting was delayed or growth of the Palmarosa was adversely affected due to cold wave during December and January. Total nitrogen, total phosphorus and total potassium in plant was estimated by Kjeldhal's method. Vandomolybdate phosphoric acid Yellow colour method in di-acid extract and Flame photometer respectively (Piper, 1966). Uptake of nutrient (N, P and K) was 
calculated by multiplying the per cent composition $(\mathrm{N}, \mathrm{P}$ and $\mathrm{K}$ ) by dry matter at each cutting.

\section{RESULTS AND DISCUSSION}

\section{Nitrogen content:}

Application of nitrogen had significant effect on the nitrogen content at each cutting. The content of nitrogen was increase with each level of nitrogen application up to $80 \mathrm{~kg} \mathrm{~N} \mathrm{ha}^{-1}$ at all three cuttings under study and the highest nitrogen content at $1^{\text {st }}, 2^{\text {nd }}$ and $3^{\text {rd }}$ cutting $(0.76$, $0.85,0.77 \%$, respectively) was recorded with the application of $80 \mathrm{~kg} \mathrm{~N} \mathrm{ha}^{-1}$ (Table 1). Whereas in $\mathrm{P}$ application significantly highest nitrogen at $1^{\text {st }}, 2^{\text {nd }}$ and $3^{\text {rd }}$ cuttings $(0.68,0.74,0.69$ per cent respectively) was recorded with the treatment $\mathrm{P}_{2}\left(40 \mathrm{~kg} \mathrm{P}_{2} \mathrm{O}_{5} \mathrm{ha}^{-1}\right)$ followed by treatment $\mathrm{P}_{1}\left(20 \mathrm{~kg} \mathrm{P}_{2} \mathrm{O}_{5} \mathrm{ha}^{-1}\right)$ (Table 1).

\section{Nitrogen uptake}

The data presented in Table 2 revealed that the nitrogen uptake was significantly influenced by various levels of nitrogen and phosphorus at different harvesting stages of crop. On an average the nitrogen uptake was found to increase from $1^{\text {st }}$ cutting to $3^{\text {rd }}$ cutting and highest uptake was recorded at $3^{\text {rd }}$ cutting.

Application of different levels of nitrogen favorably increased the nitrogen uptake at all three cuttings. Significantly highest nitrogen uptake at $1^{\text {st }}$ cutting (18 kg $\left.\mathrm{ha}^{-1}\right), 2^{\text {nd }}$ cutting $\left(16.16 \mathrm{~kg} \mathrm{ha}^{-1}\right), 3^{\text {rd }}$ cutting $\left(30.50 \mathrm{~kg} \mathrm{ha}^{-1}\right)$ was recorded under treatment $\mathrm{N}_{3}\left(80 \mathrm{~kg} \mathrm{~N} \mathrm{ha}^{-1}\right)$. In second cutting the nitrogen uptake was comparatively low, which might be due to the reduction in temperature during January, resulted into the slow growth of crop.

The application of phosphorus shown the significant effect on uptake of nitrogen at each cutting. Significantly highest nitrogen uptake was recorded at $1^{\text {st }}, 2^{\text {nd }}$ and $3^{\text {rd }}$ cuttings $\left(14.40,13.17,22.10 \mathrm{~kg} \mathrm{ha}^{-1}\right.$ respectively) under treatment $\mathrm{P}_{2}\left(40 \mathrm{~kg} \mathrm{P}_{2} \mathrm{O}_{5} \mathrm{ha}^{-1}\right)$ followed by treatment $\mathrm{P}_{1}\left(20 \mathrm{~kg} \mathrm{P}_{2} \mathrm{O}_{5}\right.$ $\left.h^{-1}\right)$.

The present findings revealed that the nitrogen uptake increased progressively with the crop growth may be due to increase in dry matter accumulation. As the dry matter accumulation increases, the nitrogen uptake in plant also increases Veerannah et al., (1976). These results also supported by Prakasha Rao et al., (1985) who reported that application of $\mathrm{N}$ fertilizers increased the uptake of $\mathrm{N}, \mathrm{P}$ and $\mathrm{K}$ by Lemon grass. These results also supported by Sundaravadivel et.al., (2000) who reported that the application of $75 \mathrm{Kg} \mathrm{N} \mathrm{ha}^{-1}$ produced highest levels of available nutrients(N,P and $\mathrm{K}$ ) in the soil.

\section{Interaction effect on Nitrogen uptake:}

Significant interaction effect of nitrogen and phosphorus on nitrogen uptake at $3^{\text {rd }}$ cutting was observed (Table 3). The highest nitrogen uptake was recorded under the treatment combination $\mathrm{N}_{3}+\mathrm{P}_{2}\left(80 \mathrm{~kg} \mathrm{~N} \mathrm{ha}^{-1}+40 \mathrm{~kg}\right.$ $\left.\mathrm{P}_{2} \mathrm{O}_{5} \mathrm{ha}^{-1}\right)$ followed by treatment $\mathrm{N}_{3}+\mathrm{P}_{1}\left(80 \mathrm{~kg} \mathrm{~N} \mathrm{ha}^{-1}+20\right.$ $\mathrm{kg} \mathrm{P}_{2} \mathrm{O}_{5}$ ha $^{-1}$ ).

\section{Phosphorus content}

The data revealed that application of nitrogen and phosphorus had significant effect on $\mathrm{P}$ content at various harvesting stages (Table 1). On an average the content of phosphorus found to increase with each cutting. The content of phosphorus was found in the range of 0.42 $0.69 \%$.

Nitrogen had significant effect on phosphorus content at each cutting. Significantly highest $\mathrm{P}$ content at $1^{\text {st }}, 2^{\text {nd }}$, and $3^{\text {rd }}$ cutting $(0.63,0.69,0.65 \%$ respectively) was recorded under treatment $\mathrm{N}_{3}\left(80 \mathrm{~kg} \mathrm{~N} \mathrm{ha}^{-1}\right)$ which is found to superior over other treatment levels (Table 1). The application of phosphorus at different levels increased the $\mathrm{P}$ content at all the harvesting stages. Significantly highest $\mathrm{P}$ content $(0.56,0.60,0.57 \%$ respectively) was recorded at $1^{\text {st }}, 2^{\text {nd }}$ and $3^{\text {rd }}$ cuttings under treatment $\mathrm{P}_{2}\left(40 \mathrm{~kg} \mathrm{P}_{2} \mathrm{O}_{5} \mathrm{ha}^{-1}\right)$ followed by treatment $\mathrm{P}_{1}\left(20 \mathrm{~kg} \mathrm{P}_{2} \mathrm{O}_{5} \mathrm{ha}^{-1}\right)$.

Interaction effect on phosphorus content

Interaction effect of nitrogen and phosphorus on $\mathrm{P}$ content was found to be non significant (Table 4).

\section{Phosphorus uptake}

It is observed from the data presented in Table 2 that significantly highest $\mathrm{P}$ uptake $\left(13.46,14.36,13.40 \mathrm{~kg} \mathrm{ha}^{-1}\right.$ respectively) at $1^{\text {st }}, 2^{\text {nd }}$ and $3^{\text {rd }}$ cuttings under treatment $\mathrm{N}_{3}$ $\left(80 \mathrm{~kg} \mathrm{~N} \mathrm{ha}^{-1}\right)$ followed by treatment $\mathrm{N}_{2}\left(60 \mathrm{~kg} \mathrm{~N} \mathrm{ha}^{-1}\right)$. The lowest $\mathrm{P}$ uptake was observed at treatment $\mathrm{N}_{0}(0 \mathrm{~kg} \mathrm{~N}$ $\left.\mathrm{ha}^{-1}\right)$.

The application of phosphorus at each level had significant effect on phosphorus uptake by Palmarosa crop at all harvesting stages. Significantly highest $P$ uptake $\left(11.40,11.90,11.40 \mathrm{~kg} \mathrm{ha}^{-1}\right.$ respectively) at $1^{\text {st }}, 2^{\text {nd }}$ and $3^{\text {rd }}$ cutting was recorded due to application of $\mathrm{P}_{2}\left(40 \mathrm{~kg} \mathrm{P}_{2} \mathrm{O}_{5}\right.$ $\left.\mathrm{ha}^{-1}\right)$, followed by treatment $\mathrm{P}_{1}\left(20 \mathrm{~kg} \mathrm{P}_{2} \mathrm{O}_{5} \mathrm{ha}^{-1}\right)$. Similar results were shown by Barooh and Khader (1990) who observed the addition of $40 \mathrm{~kg} \mathrm{P}_{2} \mathrm{O}_{5} \mathrm{ha}^{-1}$ increases significantly highest phosphorus content and stimulate biosynthesis of high energy phosphate compounds and uptake was high after 45 days of application.

\section{Interaction effect on phosphorus uptake}

The interaction effect between nitrogen and phosphorus on phosphorus uptake was found to be significant. From the data presented in Table 4 revealed that significantly highest $\mathrm{P}$ uptake was recorded under the treatment combination $\mathrm{N}_{3}+\mathrm{P}_{2}\left(80 \mathrm{~kg} \mathrm{ha}^{-1}+40 \mathrm{~kg} \mathrm{P}_{2} \mathrm{O}_{5}\right.$ ha $\left.{ }^{1}\right)$ followed by treatment combination $\mathrm{N}_{3}+\mathrm{P}_{1}(80 \mathrm{~kg} \mathrm{~N}$ ha $\left.{ }^{1}+20 \mathrm{~kg} \mathrm{P}_{2} \mathrm{O}_{5} \mathrm{ha}^{-1}\right)$.

\section{Potassium content}

It was noticed that, potassium content found to increase with each cutting and lowest was observed at $1^{\text {st }}$ cutting stage. Data presented in Table 1 revealed that, nitrogen had significant effect on $\mathrm{K}$ content at each cutting. Significantly highest K content $(0.48,0.56,0.49 \%$ respectively) was recorded due to application of $80 \mathrm{~kg} \mathrm{~N}$ $\mathrm{ha}^{-1}\left(\mathrm{~N}_{3}\right)$ followed by $60 \mathrm{~kg} \mathrm{~N} \mathrm{ha}^{-1}\left(\mathrm{~N}_{2}\right)$.

Potassium content at each cutting was found to increase with each level of phosphorus up to $40 \mathrm{~kg} \mathrm{P}_{2} \mathrm{O}_{5}$ $\mathrm{ha}^{-1}$. Significantly highest $\mathrm{K}$ content $(0.42,0.48,0.43 \%)$ was revealed under treatment $\mathrm{P}_{2}\left(40 \mathrm{~kg} \mathrm{P}_{2} \mathrm{O}_{5} \mathrm{ha}^{-1}\right)$ followed by treatment $\mathrm{P}_{1}\left(20 \mathrm{~kg} \mathrm{P}_{2} \mathrm{O}_{5} \mathrm{ha}^{-1}\right)$.

\section{Interaction effect on potassium content}

Interaction effect of nitrogen and phosphorus levels was recorded as non significant.

\section{Potassium uptake}


The data (Table 2) indicated that, the application of nitrogen had a significant effect on uptake of $\mathrm{K}$ and significantly highest $\mathrm{K}$ uptake $\left(12.70,13.25,12.96 \mathrm{~kg} \mathrm{ha}^{-1}\right.$ respectively) at $1^{\text {st }}, 2^{\text {nd }}$ and $3^{\text {rd }}$ cuttings under treatment $\mathrm{N}_{3}$ $\left(80 \mathrm{~kg} \mathrm{~N} \mathrm{ha}^{-1}\right)$ followed by treatment $\mathrm{N}_{2}\left(60 \mathrm{~kg} \mathrm{~N} \mathrm{ha}^{-1}\right)$. Whereas in case of $P$, significantly highest $K$ uptake $(9.55$, $10.12,9.82 \mathrm{~kg} \mathrm{ha}^{-1}$ respectively) at $1^{\text {st }}, 2^{\text {nd }}$ and $3^{\text {rd }}$ cuttings was recorded due to application of $40 \mathrm{~kg} \mathrm{P}_{2} \mathrm{O}_{5} \mathrm{ha}^{-1}\left(\mathrm{P}_{2}\right)$ followed by treatment $\mathrm{P}_{1}\left(20 \mathrm{~kg} \mathrm{P}_{2} \mathrm{O}_{5} \mathrm{ha}^{-1}\right)$.

These results are supported by the findings of Rajeshwara Rao et al. (1991). The reports revealed that application of
$22 \mathrm{~kg} \mathrm{P}_{2} \mathrm{O}_{5} \mathrm{ha}^{-1}$ increased $\mathrm{K}$ uptake through biomass yield of Palmarosa.

\section{Interaction effect on potassium uptake}

The interaction between nitrogen and phosphorus on uptake of $\mathrm{K}$ was found to be significant. From the data presented in interaction Table 5 revealed that significantly highest $\mathrm{K}$ uptake was recorded due to application of $80 \mathrm{~kg}$ $\mathrm{N} \mathrm{ha}^{-1}+40 \mathrm{~kg} \mathrm{P}_{2} \mathrm{O}_{5} \mathrm{ha}^{-1}\left(\mathrm{~N}_{3}+\mathrm{P}_{2}\right)$ followed by treatment combination $\mathrm{N}_{3}+\mathrm{P}_{1}$ i.e. $80 \mathrm{~kg} \mathrm{~N} \mathrm{ha}^{-1}+20 \mathrm{~kg} \mathrm{P}_{2} \mathrm{O}_{5} \mathrm{ha}^{-1}$.

Table 1: NPK contents (\%) as influenced by nitrogen and phosphorus at various cuttings

\begin{tabular}{|c|c|c|c|c|c|c|c|c|c|}
\hline & \multicolumn{3}{|c|}{ Nitrogen content $(\%)$} & \multicolumn{3}{|c|}{ Phosphorus content $(\%)$} & \multicolumn{3}{|c|}{ Potassium content $(\%)$} \\
\hline & $\begin{array}{l}\mathbf{1}^{\text {st }} \\
\text { cutting }\end{array}$ & $\begin{array}{l}2^{\text {nd }} \\
\text { cutting }\end{array}$ & $\begin{array}{l}3^{\text {rd }} \\
\text { cutting }\end{array}$ & $\begin{array}{l}1^{\text {st }} \\
\text { cutting }\end{array}$ & $\begin{array}{l}2^{\text {nd }} \\
\text { cutting }\end{array}$ & $\begin{array}{l}3^{\text {rd }} \\
\text { cutting }\end{array}$ & $\begin{array}{l}1^{\text {st }} \\
\text { cutting }\end{array}$ & $\begin{array}{l}2^{\text {nd }} \\
\text { cutting }\end{array}$ & $\begin{array}{l}3^{\text {rd }} \\
\text { cutting }\end{array}$ \\
\hline \multicolumn{10}{|c|}{ Nitrogen levels $\left(\mathrm{kg} \mathrm{ha}^{-1}\right)$} \\
\hline $\mathrm{N}_{0}-0$ & 0.52 & 0.55 & 0.53 & 0.42 & 0.47 & 0.43 & 0.32 & 0.35 & 0.33 \\
\hline $\mathrm{N}_{1}-40$ & 0.65 & 0.69 & 0.66 & 0.52 & 0.56 & 0.54 & 0.37 & 0.42 & 0.37 \\
\hline $\mathrm{N}_{2}-60$ & 0.73 & 0.77 & 0.74 & 0.59 & 0.62 & 0.60 & 0.44 & 0.49 & 0.44 \\
\hline $\mathrm{N}_{3}-80$ & 0.76 & 0.85 & 0.77 & 0.63 & 0.69 & 0.65 & 0.48 & 0.56 & 0.49 \\
\hline 'F' test & $\mathrm{S}$ & $\mathrm{S}$ & $\mathrm{S}$ & $\mathrm{S}$ & $\mathrm{S}$ & $\mathrm{S}$ & $\mathrm{S}$ & $\mathrm{S}$ & $\mathrm{S}$ \\
\hline $\mathrm{SE}(\mathrm{m}) \pm$ & 0.005 & 0.008 & 0.008 & 0.009 & 0.006 & 0.007 & 0.005 & 0.008 & 0.005 \\
\hline $\mathrm{CD}$ at $5 \%$ & 0.015 & 0.023 & 0.024 & 0.02 & 0.016 & 0.019 & 0.015 & 0.023 & 0.013 \\
\hline \multicolumn{10}{|c|}{ Phosphorus levels $\left(\mathrm{kg} \mathrm{ha}^{-1}\right)$} \\
\hline $\mathrm{P}_{0}-0$ & 0.65 & 0.69 & 0.66 & 0.53 & 0.56 & 0.53 & 0.38 & 0.43 & 0.39 \\
\hline$P_{1}-20$ & 0.66 & 0.71 & 0.67 & 0.54 & 0.58 & 0.56 & 0.40 & 0.45 & 0.40 \\
\hline$P_{2}-40$ & 0.68 & 0.74 & 0.69 & 0.56 & 0.60 & 0.57 & 0.42 & 0.48 & 0.43 \\
\hline 'F' test & $\mathrm{S}$ & $\mathrm{S}$ & S & $\mathrm{S}$ & $\mathrm{S}$ & $\mathrm{S}$ & $\mathrm{S}$ & $\mathrm{S}$ & $\mathrm{S}$ \\
\hline $\mathrm{SE}(\mathrm{m}) \pm$ & 0.004 & 0.007 & 0.007 & 0.008 & 0.005 & 0.006 & 0.004 & 0.007 & 0.004 \\
\hline $\mathrm{CD}$ at $5 \%$ & 0.013 & 0.020 & 0.020 & 0.022 & 0.014 & 0.017 & 0.013 & 0.020 & 0.013 \\
\hline \multicolumn{10}{|l|}{ Interaction } \\
\hline 'F' test & NS & NS & $\mathrm{S}$ & NS & NS & NS & NS & NS & NS \\
\hline $\begin{array}{l}\mathrm{SE}(\mathrm{m}) \pm \\
\mathrm{CD} \text { at } 5 \%\end{array}$ & 0.0028 & 0.0042 & 0.0044 & 0.0046 & 0.0030 & 0.0036 & 0.0027 & 0.0043 & 0.0028 \\
\hline
\end{tabular}

(S: Significant and NS: Non significant)

Table 2: Effect of nitrogen and phosphorus on N, P and $K$ uptake $\left(\mathrm{kg} \mathrm{ha}^{-1}\right)$ at various cuttings

\begin{tabular}{|c|c|c|c|c|c|c|c|c|c|}
\hline & \multicolumn{3}{|c|}{ Nitrogen uptake $\left(\mathrm{Kg} \mathrm{ha}^{-1}\right)$} & \multicolumn{3}{|c|}{ Phosphorus uptake $\left(\mathrm{Kg} \mathrm{ha}^{-1}\right)$} & \multicolumn{3}{|c|}{ Potassium uptake $\left(\mathrm{Kg} \mathrm{ha}^{-1}\right)$} \\
\hline & $\begin{array}{l}1^{\text {st }} \\
\text { cutting }\end{array}$ & $\begin{array}{l}2^{\text {nd }} \\
\text { cutting }\end{array}$ & $\begin{array}{l}3^{\text {rd }} \\
\text { cutting }\end{array}$ & $\begin{array}{l}\mathbf{1}^{\text {st }} \\
\text { cutting }\end{array}$ & $\begin{array}{l}2^{\text {nd }} \\
\text { cutting }\end{array}$ & $\begin{array}{l}3^{\text {rd }} \\
\text { cutting }\end{array}$ & $\begin{array}{l}1^{\text {st }} \\
\text { cutting }\end{array}$ & $\begin{array}{l}2^{\text {nd }} \\
\text { cutting }\end{array}$ & $\begin{array}{l}3^{\text {rd }} \\
\text { cutting }\end{array}$ \\
\hline \multicolumn{10}{|c|}{ Nitrogen levels $\left(\mathrm{kg} \mathrm{ha}^{-1}\right)$} \\
\hline $\mathrm{N}_{0}-0$ & 8.24 & 8.93 & 11.90 & 7.37 & 7.50 & 7.40 & 5.23 & 5.80 & 5.46 \\
\hline $\mathrm{N}_{1}-40$ & 12.70 & 10.90 & 15.93 & 9.81 & 10.00 & 9.83 & 7.23 & 7.97 & 7.56 \\
\hline$N_{2}-60$ & 15.92 & 13.50 & 22.46 & 12.64 & 13.20 & 12.25 & 9.86 & 10.33 & 10.10 \\
\hline $\mathrm{N}_{3}-80$ & 18.00 & 16.16 & 30.50 & 13.46 & 14.36 & 13.40 & 12.70 & 13.25 & 12.96 \\
\hline 'F' test & $\mathrm{S}$ & $\mathrm{S}$ & $\mathrm{S}$ & $\mathrm{S}$ & $\mathrm{S}$ & $\mathrm{S}$ & $\mathrm{S}$ & $\mathrm{S}$ & $\mathrm{S}$ \\
\hline $\mathrm{SE}(\mathrm{m}) \pm$ & 0.31 & 0.14 & 0.09 & 0.07 & 0.08 & 0.15 & 0.098 & 0.096 & 0.040 \\
\hline $\mathrm{CD}$ at $5 \%$ & 0.88 & 0.39 & 0.25 & 0.220 & 0.227 & 0.43 & 0.27 & 0.26 & 0.13 \\
\hline \multicolumn{10}{|c|}{ Phosphorus levels $\left(\mathrm{kg} \mathrm{ha}^{-1}\right)$} \\
\hline $\mathrm{P}_{0}-0$ & 12.70 & 11.60 & 18.40 & 10.34 & 10.72 & 10.00 & 7.72 & 8.32 & 8.00 \\
\hline$P_{1}-20$ & 14.00 & 12.30 & 20.10 & 10.72 & 11.17 & 10.77 & 9.00 & 9.60 & 9.25 \\
\hline$P_{2}-40$ & 14.40 & 13.17 & 22.12 & 11.40 & 11.90 & 11.40 & 9.55 & 10.12 & 9.82 \\
\hline 'F' test & $\mathrm{S}$ & $\mathrm{S}$ & $\mathrm{S}$ & $\mathrm{S}$ & $\mathrm{S}$ & $\mathrm{S}$ & $\mathrm{S}$ & $\mathrm{S}$ & $\mathrm{S}$ \\
\hline $\mathrm{SE}(\mathrm{m}) \pm$ & 0.27 & 0.12 & 0.07 & 0.06 & 0.07 & 0.13 & 0.084 & 0.083 & 0.040 \\
\hline $\mathrm{CD}$ at $5 \%$ & 0.76 & 0.34 & 0.22 & 0.19 & 0.19 & 0.37 & 0.25 & 0.23 & 0.11 \\
\hline Interaction & & & & & & & & & \\
\hline 'F' test & NS & NS & $\mathrm{S}$ & $\mathrm{S}$ & $\mathrm{S}$ & $\mathrm{S}$ & $\mathrm{S}$ & $\mathrm{S}$ & $\mathrm{S}$ \\
\hline
\end{tabular}


Journal of Progressive Agriculture, Vol.3, No. 2: Oct. 2012

\begin{tabular}{|c|c|c|c|c|c|c|c|c|c|}
\hline $\begin{array}{l}\mathrm{SE}(\mathrm{m}) \pm \\
\mathrm{CD} \text { at } 5 \%\end{array}$ & 0.049 & 0.048 & $\begin{array}{l}0.04 \\
0.12\end{array}$ & $\begin{array}{l}0.03 \\
0.110\end{array}$ & $\begin{array}{l}0.04 \\
0.113\end{array}$ & $\begin{array}{l}0.07 \\
0.210\end{array}$ & $\begin{array}{l}0.049 \\
0.135\end{array}$ & $\begin{array}{l}0.048 \\
0.134\end{array}$ & $\begin{array}{l}0.020 \\
0.066\end{array}$ \\
\hline
\end{tabular}

(S: Significant and NS: Non significant)

Table 3: Interaction effect of nitrogen and phosphorus on nitrogen uptake $\left(\mathrm{kg} \mathrm{ha}^{-1}\right)$ at third cutting

\begin{tabular}{|c|c|c|c|c|}
\hline \multirow[t]{2}{*}{ Treatments } & \multicolumn{3}{|c|}{ Phosphorus levels kg ha ${ }^{-1}$} & \multirow[b]{2}{*}{$\operatorname{Mean}(\mathbf{N})$} \\
\hline & $\mathbf{P}_{\mathbf{0}}$ & $\mathbf{P}_{1}$ & $\mathbf{P}_{2}$ & \\
\hline \multicolumn{5}{|c|}{ Nitrogen levels $\left(\mathrm{kg} \mathrm{ha}^{-1}\right)$} \\
\hline $\mathrm{N}_{0}$ & 10.50 & 11.80 & 13.50 & 11.90 \\
\hline $\mathrm{N}_{1}$ & 14.80 & 15.90 & 17.10 & 15.93 \\
\hline $\mathrm{N}_{2}$ & 20.50 & 22.50 & 24.40 & 22.46 \\
\hline $\mathrm{N}_{3}$ & 27.80 & 30.20 & 33.50 & 30.50 \\
\hline Mean $(\mathrm{P})$ & 18.40 & 20.10 & 22.12 & - \\
\hline 'F' test & $\mathrm{S}$ & & & \\
\hline $\mathrm{SE}(\mathrm{m}) \pm$ & 0.04 & & & \\
\hline $\mathrm{CD}$ at $5 \%$ & 0.12 & & & \\
\hline
\end{tabular}

(S: Significant and NS: Non significant)

Table 4: Interaction effect of nitrogen and phosphorus on phosphorus uptake $\left(\mathrm{kg} \mathrm{ha}^{-1}\right)$ at various cuttings

\begin{tabular}{|c|c|c|c|c|c|c|c|c|c|c|c|c|}
\hline \multicolumn{5}{|c|}{$1^{\text {st }}$ cutting } & \multicolumn{3}{|c|}{$2^{\text {nd }}$ cutting } & \multicolumn{4}{|c|}{$3^{\text {rd }}$ cutting } & \multirow[b]{2}{*}{$\begin{array}{l}\text { Mean } \\
(\mathrm{N})\end{array}$} \\
\hline & $\mathrm{P}_{0}$ & $\mathrm{P}_{1}$ & $\mathrm{P}_{2}$ & $\begin{array}{l}\text { Mean } \\
(\mathrm{N})\end{array}$ & $\mathrm{P}_{0}$ & $\mathrm{P}_{1}$ & $\mathrm{P}_{2}$ & $\begin{array}{l}\text { Mean } \\
(\mathrm{N})\end{array}$ & $\mathrm{P}_{0}$ & $\mathrm{P}_{1}$ & $\mathrm{P}_{2}$ & \\
\hline $\mathrm{N}_{0}$ & 6.33 & 7.16 & 8.63 & 7.37 & 6.50 & 7.50 & 8.50 & 7.50 & 6.40 & 7.20 & 8.60 & 7.40 \\
\hline $\mathrm{N}_{1}$ & 9.40 & 9.63 & 10.40 & 9.81 & 9.50 & 9.70 & 10.80 & 10.00 & 9.40 & 9.60 & 10.50 & 9.83 \\
\hline $\mathrm{N}_{2}$ & 12.53 & 12.60 & 12.80 & 12.64 & 12.90 & 13.20 & 13.50 & 13.20 & 11.00 & 12.80 & 12.90 & 12.25 \\
\hline $\mathrm{N}_{3}$ & 13.10 & 13.50 & 13.80 & 13.46 & 14.00 & 14.30 & 14.80 & 14.36 & 13.20 & 13.50 & 13.60 & 13.40 \\
\hline Mean $(\mathrm{P})$ & 10.34 & 10.72 & 11.40 & - & 10.72 & 11.17 & 11.90 & - & 10.00 & 10.77 & 11.40 & - \\
\hline 'F' test & S & & & & S & & & & S & & & \\
\hline $\mathrm{SE}(\mathrm{m}) \pm$ & 0.03 & & & & 0.04 & & & & 0.07 & & & \\
\hline $\begin{array}{l}\mathrm{CD} \text { at } 5 \\
\%\end{array}$ & 0.110 & & & & 0.113 & & & & 0.21 & & & \\
\hline
\end{tabular}

$\%$

(S: Significant and NS: Non significant)

Table 5: Interaction effect of nitrogen and phosphorus on potassium uptake $\left(\mathrm{kg} \mathrm{ha}^{-1}\right)$ at various cuttings

\begin{tabular}{|c|c|c|c|c|c|c|c|c|c|c|c|c|}
\hline \multicolumn{5}{|c|}{$1^{\text {st }}$ cutting } & \multicolumn{3}{|c|}{$2^{\text {nd }}$ cutting } & \multicolumn{5}{|c|}{$3^{\text {rd }}$ cutting } \\
\hline & $\mathrm{P}_{0}$ & $\mathrm{P}_{1}$ & $\mathrm{P}_{2}$ & $\begin{array}{l}\text { Mean } \\
(\mathrm{N})\end{array}$ & $\mathrm{P}_{0}$ & $\mathrm{P}_{1}$ & $\mathrm{P}_{2}$ & $\begin{array}{l}\text { Mean } \\
(\mathrm{N})\end{array}$ & $\mathrm{P}_{0}$ & $\mathrm{P}_{1}$ & $\mathrm{P}_{2}$ & $\begin{array}{l}\text { Mean } \\
(\mathrm{N})\end{array}$ \\
\hline $\mathrm{N}_{0}$ & 4.70 & 5.40 & 5.60 & 5.23 & 5.00 & 6.00 & 6.50 & 5.83 & 4.90 & 5.60 & 5.90 & 5.46 \\
\hline $\mathrm{N}_{1}$ & 5.90 & 7.50 & 8.30 & 7.23 & 7.00 & 8.00 & 8.93 & 7.97 & 6.30 & 7.80 & 8.60 & 7.56 \\
\hline $\mathrm{N}_{2}$ & 9.00 & 9.90 & 10.70 & 9.86 & 9.50 & 10.50 & 11.00 & 10.33 & 9.30 & 10.10 & 10.90 & 10.10 \\
\hline $\mathrm{N}_{3}$ & 11.30 & 13.20 & 13.60 & 12.70 & 11.80 & 13.90 & 14.05 & 13.25 & 11.50 & 13.50 & 13.90 & 12.96 \\
\hline $\begin{array}{l}\text { Mean } \\
(\mathrm{P})\end{array}$ & 7.72 & 9.00 & 9.50 & - & 8.32 & 9.60 & 10.12 & - & 8.00 & 9.25 & 9.82 & - \\
\hline 'F' test & $\mathrm{S}$ & & & & $\mathrm{S}$ & & & & $\mathrm{S}$ & & & \\
\hline $\begin{array}{l}\text { SE (m) } \\
\pm\end{array}$ & 0.049 & & & & 0.048 & & & & 0.24 & & & \\
\hline $\begin{array}{l}\mathrm{CD} \\
5 \%\end{array}$ & 0.135 & & & & 0.134 & & & & 0.066 & & & \\
\hline
\end{tabular}

(S: Significant and NS: Non significant)

\section{REFERENCES}

Barooh, M. and Khader, M.D. 1990. Studies on phosphorus utilization by Palmarosa (Cymgopogon martinii var. Motia). Indian Perfumer. 34(2): 147-151.

Mallavarapu, G.R., Rajeswara Rao, B.R., Kaul, P.N., Ramesh, S. and Bhattacharya, A.K., 1998. Volatile constituents of the essential oils of the seeds and herb of palmarosa (Cymbopogon martinii (Roxb.) Wats. var. motia Burk.). Flavour Fragrance J. 13: 167-169.

Pareek, S. K., Maheswari, M. L., Singh K. D. and R. Gupta. 1983. Nutrient uptake and dry matter production of 
Palmarosa oil grass under different levels of N, P and $\mathrm{K}$ fertilizers. International Journal for Tropical Agriculture 1(3): 203-09.

Piper, C.S. 1966. Soil and plant analysis $4^{\text {th }}$ edition Inter Science Publishers, New York.

Prakasa Rao, E.V.S., Singh, M., Ganesha Rao, R.S., Rajeswara Rao, B.R., 1985. Effect of plant spacing and application of nitrogen fertilizer on herb and essential oil yields of palmarosa (Cymbopogon martinii Stapf. var. motia). J. Agric. Sci. Camb. 104: 67-70.

Prakasha Rao, E.V.S., Singh, M. Ganesh Rao, R.S. 1985. Effect of N, P and $K$ fertilizers on yield and nutrient uptake of lemongrass (Cymbopogon flexuosus). Int. J. Crop Agric. 3(2): 123-127.

Rajeshwara Rao, B. R., Prakasa Rao, E. V. S., Singh, K. Singh, M., Kaul, P. N. and Bhattacharya, A. K.
1991. Fertilizer effect on Palmarosa (Cymbopogon martinii) under semi-arid tropical conditions of India. Indian Journal for Agricultural Sciences. 61(7): 169-174.

Rajeswara Rao, B.R., 1999. Aromatic plants for dry areas. In:Singh, R.P., Osman, M. (Eds.), Sustainable Alternate Land Use Systems for Drylands. Oriental Enterprises, Dehradun,India,pp.157-170.

Sundaravadivel, K., T. Chitdeswari, M. Periswamy, M. Subrahamanian and D. Krishnadas. 2000. Nitrogen levels and sources on herb oil yield and soil fertility of rainfed Palmarosa. Madras Agric. J. 86(4-6) : 272-274.

Veerannah, L., Balakrishnna, R. and Rangaswamy, M. 1976. Studies on pattern of nutrient uptake in medicinal and aromatic plants. Annual Report on MAP. pp 94-98. 